\title{
A spontaneously sustainable bioinspired interfacial evaporation driven power generation strategy
}

\author{
Zhuangzhi Sun ( $\nabla$ sunzhuangzhi@nefu.edu.cn ) \\ Northeast Forestry University https://orcid.org/0000-0003-0028-2426 \\ Zhaoxin Li \\ Northeast Forestry University \\ Changlong Han \\ Northeast Forestry University \\ Mingxing Jing \\ Northeast Forestry University \\ Haipeng Yu \\ Northeast Forestry University \\ Zuankai Wang \\ City University of Hong Kong https://orcid.org/0000-0002-3510-1122
}

\section{Article}

Keywords: interfacial evaporation driven nanogenerator, liquid evaporation, bionic light-trapping

Posted Date: August 10th, 2021

DOI: https://doi.org/10.21203/rs.3.rs-753838/v1

License: (c) (i) This work is licensed under a Creative Commons Attribution 4.0 International License. Read Full License

Version of Record: A version of this preprint was published at Nature Communications on August 29th, 2022. See the published version at https://doi.org/10.1038/s41467-022-32820-0. 


\title{
A spontaneously sustainable bioinspired interfacial evaporation driven power generation strategy
}

\author{
Zhuangzhi Sun ${ }^{1,2, *}$, Zhaoxin Li ${ }^{1, *}$, Chuanlong Han ${ }^{1}$, Mingxing Jing ${ }^{1}$, HaipengYu ${ }^{2, *}$, Zuankai Wang,"* \\ ${ }^{1}$ Province Key Laboratory of Forestry Intelligent Equipment Engineering, College of Mechanical and Electrical \\ Engineering, Northeast Forestry University, Harbin 150000, People's Republic of China. \\ ${ }^{2}$ Key Laboratory of Bio-based Material Science \& Technology, Ministry of Education, Northeast Forestry \\ University, Harbin 150000, People's Republic of China. \\ ${ }^{3}$ Department of Mechanical and Biomedical Engineering, City University of Hong Kong, Hong Kong, People's \\ Republic of China. \\ E-mail: sunzhuangzhi@nefu.edu.cn; 2014211213@nefu.edu.cn; yuhaipeng20000@nefu.edu.cn; \\ zuanwang@ cityu.edu.hk
}

In nature, liquid evaporation occurs everywhere all the time. This low-grade energy absorption to drive liquid evaporation is greatly potential for sustainable spontaneously power generation. Here, a natural liquid evaporation strategy of interfacial evaporation driven nanogenerator (IENG) is developed in this work. Coupled by the phonon wind and a fluctuating Coulomb field, an induced direct current is generated. Simultaneously, inspired by the light-trapping properties of moth eye, a simple and efficient BLT-IENG including a hierarchical surface of bionic light-trapping and electrospinning perovskite conductivity with an enhanced thermally insulating and water storage capability is designed. This enhancement of the output performance is greatly attributed to the improvement of the interfacial evaporation characteristics driven by natural solar and wind energies. Hence, our BLT-IENG achieves a breakthrough in the unit area open-circuit voltage in the marine environment, which is improved by a factor of 7.6 over the currently reported average value. This work provides an unexplored strategy for multi-energies inspired natural interfacial evaporation driven power generation. 


\section{Introduction}

In nature, liquid evaporation occurs everywhere all the time when water is present and exposed 1-3. Compared to boiling, the required threshold for water evaporation is very low. Among them, interfacial evaporation represents the vaporization of water molecules on a solid-liquid surface or a gas-liquid surface ${ }^{4,5}$. The temperature, surface area of the liquid, and airflow rate on the liquid surface are essential factors for the speed of interfacial evaporation 6,7. As a normal natural phenomenon, interfacial evaporation provides many inspirations to the sustainable spontaneously power generation. Therefore, the tentative exploration of the simple, natural interfacial evaporation driven power generation strategy is very technically promising.

Although the photothermal conversion characteristics are still the limiting bottleneck of the interfacial evaporation performance, the functional structure of natural organisms provides us with new inspiration ${ }^{8-11}$. In view of the interfacial light and heat absorption of solar energy, the lighttrapping structure on the surface of moth eyes gives us much inspiration due to its zero reflection of sunlight ${ }^{12-14}$. The all-inorganic-type perovskite $\left(\mathrm{Cs}_{4} \mathrm{PbBr}_{6}\right)$ with a crystal structure similar to the moth eye structure exhibits a wide light absorption spectrum, achieving effective absorption of sunlight ${ }^{15,16}$. Moreover, interfacial evaporation also largely depends on the water supply/ storage and thermal energy retention ${ }^{17,18}$. In addition, the use of natural wind to accelerate the airflow velocity at the surface liquid will provide effective support for interfacial evaporation ${ }^{19,20}$.

Here, we developed a simple and efficient interfacial evaporation driven nanogenerator (BLTIENG) including a surface bionic light-trapping and conductivity (BLC) structure and a thermal insulating and water storage (ITW) structure driven by solar and wind energies. The bionic lighttrapping structure is transplanted to the surface of the BLT-IENG by using the 3D template method. The electrospinning photothermal conductivity layer with light-absorbing particles of $\mathrm{Cs}_{4} \mathrm{PbBr}_{6}$ type perovskite/ MWNTs is sprayed on the bionic light-trapping structure surface by a layer-bylayer self-assembly method to construct the BLC-layer. At the same time, the ITW-layer is designed by using enhanced thermally insulating particles of ionic liquid (1-n-butyl-3-methylimidazolium chloride $(\mathrm{BMIMCl}))$ /cellulose/ titanium dioxide $\left(\mathrm{TiO}_{2}\right) /$ silica $\left(\mathrm{SiO}_{2}\right)$. Our BLT-IENG shows excellent interfacial evaporation characteristics, including high light absorption efficiency (96.7\%), low thermal conductivity $\left(0.766 \mathrm{~W} \mathrm{~m}^{-1} \mathrm{~h}^{-1}\right)$, large thermal insulating capacity (increased by 1.5 times), excellent evaporation rate $\left(2.78 \mathrm{~kg} \mathrm{~m}^{-2} \mathrm{~h}^{-1}\right)$ and outstanding energy conversion efficiency $(97.9 \%)$ under a light intensity of $1.0 \mathrm{~kW} \cdot \mathrm{m}^{-2}$. Hence, our BLT-IENG exhibits a short-circuit current of $222.07 \mu \mathrm{A}$, an output power density of $34.15 \mu \mathrm{W} \mathrm{cm} \mathrm{cm}^{-2}$, and a unit area open-circuit voltage of $276 \mathrm{mV} \mathrm{cm} \mathrm{m}^{-2}$ in the marine environment with a light intensity of $2.0 \mathrm{~kW} \cdot \mathrm{m}^{-2}$ and a wind speed of $1 \mathrm{~m} \mathrm{~s}^{-1}$. This demonstrates a new idea to develop natural interfacial evaporation driven 
power generation systems and provides a new attempt to obtain multiple energies harvesting from natural environments.

\section{Results}

\section{Structure design and characterization analysis of the BLT-IENG}

As conceptually shown in Fig. 1a, the bionic light-trapping structure was prepared on the surface of the BLT-IENG by 3D printing based on the microscopic size and array structure of the moth eye. Then, its surface was covered with a high-efficiency $\mathrm{Cs}_{4} \mathrm{PbBr}_{6}$ photothermal conductivity substrate by electrospinning to prepare the BLC-layer. Meanwhile, an ionic liquid (BMIMCl), nanoparticles $\left(\mathrm{TiO}_{2} / \mathrm{SiO}_{2}\right)$ and $\alpha$-cellulose were uniformly dispersed in the thermal insulation substrates to construct the ITW-layer (Supplementary Fig. 1). Our BLT-IENG exhibits efficient solid-liquid interfacial evaporation characteristics for the power generation. With the action of interfacial evaporation driven by solar and wind energies, a flowing potential is formed by the accumulated pressure difference in the multiwalled carbon nanotube (MWNT)-microchannels. After connection, electrons move along the wire from the wet end at the bottom to the dry end exposed to air by the coupling action of the phonon wind and a fluctuating Coulomb field, and an induced direct current is finally generated.

The bean hawk moth eyes are selected as the experimental object in this work (Supplementary Fig. 2a). The inside of the moth eye is composed of a hexagonal structure (Supplementary Fig. 2b). The partial enlargement shows that the inside of the hexagon is formed by an array of cone-like structures (Fig. 1b). After partial extraction, mesopores with a diameter of approximately $20 \mathrm{~nm}$ are dispersed in the BLT-IENG (Supplementary Fig. 2c). The bionic moth eye structure was fabricated and screened by the 3D template method (Fig. 1c, Supplementary Fig. 3 and Supplementary Fig. 4). The experimental samples with the BLC-layer (Fig. 1d) were prepared by surface spraying nanocomposite materials (Cs4PbBr6, MWNTs, etc.) (Supplementary Fig. 5). The BLC-layer with the $\mathrm{Cs}_{4} \mathrm{PbBr}_{6}$-type perovskite enhances the light absorption efficiency and stability by changing the reflection or refraction light path. The SEM image shows that the surface of the perovskite is composed of a massive structure (Supplementary Fig. 2d), and a cone-like structure is presented in the TEM image (Fig. 1a), whose shape is close to that of the moth eye.

\section{Power generation performance of the BLT-IENG induced by the interfacial evaporation}

Studies show that under the light intensity of $2 \mathrm{~kW} \cdot \mathrm{m}^{-2}$ and the wind speed of $1 \mathrm{~m} \cdot \mathrm{s}^{-1}$, the open circuit voltage of the BLT-IENG is as high as $1.106 \mathrm{~V}$ (Fig. 2a), and the short-circuit current is as high as $222.067 \mu \mathrm{A}$ (Fig. 2b). By comparison with Control 2, it is found that the design of the BLTIENG with bionic light-trapping structure can effectively increase its output voltage and output 
current by nearly 4 times. The power generation performance of the BLT-IENG is closely related to the interface conductivity and the evaporation capacity. The strength of the electrical signal generated by Control 1 is related to the conductive properties of the Ti derivatives presented in the evaporation interface. The open circuit voltage and the short-circuit current of INEG (Control 2) after MWNTs composite enhancement with high conductivity has been significantly improved by nearly 3 times and 8 times than Control 1 . In order to eliminate the interference of external factors, the power generation performance parameters of the interfacial evaporation of pure $\alpha$-cellulose was experimented in a nearly vacuum environment. The transient current and voltage signals are very weak, which is almost close to zero (Supplementary Fig. 6c, d). Fig. 2c and Fig. 2d show in detail the voltage and current changes produced by the BLT-IENG during the interfacial evaporation inspired power generation process. When the evaporation rate of the BLT-IENG gradually rises to a stable maximum value, the water absorption and evaporation loss reach a dynamic equilibrium state, which makes the voltage and current generated by the BLT-IENG during this process show corresponding dynamic changes. This process effectively reflects the relationship between the BLT-IENG's power generation and water evaporation. The excellent power generation of the BLTIENG is mainly attributed to its evaporation performance under the BLC-layer and the ITW-layer (Fig. 2e). The water evaporation rate of the BLT-IENG $\left(2.78 \mathrm{~kg} \mathrm{~m}^{-2} \mathrm{~h}^{-1}\right)$ is greatly improved compared to the original IENGs $\left(1.848 \mathrm{~kg} \mathrm{~m}^{-2} \mathrm{~h}^{-1}\right)$, and it is also higher than some existing reports about solar evaporators (Supplementary Fig. 7a and Supplementary Note 1). In addition, the energy conversion efficiency of the BLT-IENG $(97.8 \%)$ is also greatly improved compared to the original IENGs (60.2\%), and is higher than other reported solar evaporators (Supplementary Fig. 7b and Supplementary Note 2).

\section{Coupling enhancement effect on interfacial evaporation of the BLT-IENG}

The light absorbance of the BLT-IENG with the BLC-layer was measured at wavelengths of 190-2500 nm (Fig. 3a). The average light absorption efficiency is approximately $94.7 \%$, which is due to the angle of incidence being reduced by the bionic light-trapping structure. The 3D printing manufacturing process method for the BLT-IENG with the bionic light-trapping structure leads to the best photothermal characteristics (Supplementary Fig. 8a). According to the equations given below, the theoretical reflectivity is $3 \%$ (the theoretical model, see Supplementary Fig. 8b) ${ }^{23}$.

$$
\begin{gathered}
f(x, y)=\left\{\begin{array}{cc}
0 & x^{2}+y^{2}>d \\
n_{2}-n_{1} & x^{2}+y^{2} \leq d
\end{array}\right. \\
F(\mathrm{x}, \mathrm{y})=n_{1}+f(x, y) *\left[\operatorname{comb}\left(\frac{x}{\Lambda}\right) \operatorname{comb}\left(\frac{y}{\Lambda}\right)\right]
\end{gathered}
$$


where $n_{2}$ is the refractive index of the substrate, $n_{1}$ is the refractive index of air, $d$ is the diameter of the cylinder, $f(\mathrm{x}, \mathrm{y})$ is a single periodic function, and $*$ is the convolution symbol. The refractive index of the moth eye structure is $n_{2}=30$ in the long-wave infrared band. The microstructure period $\Lambda$ is $(50 \pm 0.1) \mu \mathrm{m}$, depth $h$ is $(30 \pm 0.5) \mu \mathrm{m}$, and bottom diameter $d$ is $(20 \pm 0.1) \mu \mathrm{m}$.

This bionic light-trapping structure can reflect and transmit light multiple times, thereby increasing the absorption efficiency of the sunlight. After adding perovskite on the surface, the gaps are filled. The light absorption effect and light absorption stability are further improved (Supplementary Note 3 and Fig. 3a). This results in an average absorption efficiency of approximately $96.8 \%$, which is higher than that of other types of solar evaporators (Supplementary Fig. 8c). Considering the theoretical reflectance values of the moth eyes, the similarity between the bionic moth eye structure and the moth eye structure is confirmed from the light absorption. The area enclosed by the white dashed line in Fig. 3a is the stable absorption area of the BLT-IENG with the BLC-layer. Its average difference is approximately $2 \%$, which is lower than the average light absorption efficiency difference of the reported solar evaporators in the stable phase (Supplementary Fig. 8d). According to the photothermal characteristic results, the surface temperature (Fig. 3b and Supplementary Fig. 9) are significantly improved, and the thermal conductivity $\left(0.766 \mathrm{~W} \cdot \mathrm{m}^{-1} \cdot \mathrm{k}^{-1}\right)($ Fig. $3 \mathrm{c}$ and Supplementary Note 4$)$ of the BLT-IENG has a certain degree of increase.

Simultaneously, experiments show that the BLC-layer surface has a high thermal conductivity, while the thermal conductivity of the ITW-layer is very low $\left(0.743 \mathrm{Wm}^{-1} \mathrm{k}^{-1}\right)$. The $\mathrm{TiO}_{2}$ and $\mathrm{SiO}_{2}$ particles inside can reflect not only light but also heat radiation. Thus, the reflected heat radiation can diffuse back to the inside, thereby storing the absorbed heat converted from light in the ITWlayer. This improves the overall temperature of the BLT-IENG system (Supplementary Fig. 10a). Comparing the surface temperature of different ITW-layer, the thermal insulation performance of the ITW-layers with $\mathrm{TiO}_{2}$ or $\mathrm{SiO}_{2}$ is greatly improved (Supplementary Fig. 10b). Among them, the surface temperature of the ITW-layer with $\mathrm{TiO}_{2}$ and $\mathrm{SiO}_{2}$ is basically stable, which is approximately $30{ }^{\circ} \mathrm{C}$ after $1 \mathrm{~h}$ of evaporation (Fig. 3d). Meanwhile, the water absorption capacity of the ITW-layer can be expressed by its water content $(Q)$ in the saturated state, which is represented by $Q=\left(m_{1^{-}}\right.$ $\left.m_{2}\right) / m_{1}{ }^{24}$, where $m_{1}$ is the weight of the ITW-layer in the saturated state $(\mathrm{g})$ and $m_{2}$ is the weight of the ITW layer after drying treatment $(\mathrm{g})$. Result shows that the highest water content ratio is approximately $82 \%$ (Supplementary Fig. 10c).

In general, the temperature gradient of the BLC-layer in the natural environment after illuminated by the sunlight is relatively large, and relatively speaking, the temperature of the ITW-layer can be maintained stably (Fig. 3e). The BLT-IENG with the light-trapping structure, whose upper surface 
is covered with the photothermal BLC-layer and lower surface existing thermal insulating ITWlayer can achieve efficient interface photothermal conversion (Fig. 3f). A large temperature shock is generated at the evaporation interface to achieve efficient interface photothermal conversion, and the moisture storage maintains good thermal insulating properties to ensure a stable moisture supply.

The coupling enhancement effect of interfacial evaporation of BLT-IENG can be summarized as follows. The $\mathrm{BMIM}^{+}$cations and water molecules are fixed through intermolecular forces and hydrogen bonds in the BLT-IENG. A small portion of water molecules is converted into bound water and intermediate water by $\alpha$-cellulose via hydrogen bonds, but most water molecules are free water (Supplementary Fig. 11a). The bound water and intermediate water can weaken the van der Waals forces among the water molecules in the BLT-IENG. Thus, the enthalpy change in the water evaporation is reduced, thereby increasing the water evaporation rate and the energy conversion efficiency. Moreover, the BLT-IENG with the perovskite $\left(\mathrm{Cs}_{4} \mathrm{PbBr}_{6}\right)$ reaches a high vibrational energy level by absorbing photons. When it returns to the lowest vibrational energy level, the energy difference is released in the form of heat, which makes the overall temperature rise and accelerates the interfacial evaporation.

Meanwhile, the electrons and holes in $\mathrm{TiO}_{2}$, $\mathrm{TiNx}$ and $\mathrm{SiO}_{2}$ particles constitute carriers in the semiconductor. In actual situations, these electrons are easily affected by various scattering factors, causing them to transit between different electronic states. When the system is in dynamic equilibrium, there will be many electron-hole pairs in the particles. When the BLT-IENG is stimulated by the sunlight, the concentration of electrons and holes will change due to changes in external conditions, and the system will no longer maintain a dynamic balance. In the case of nonequilibrium, the excess carriers will recombine. When the external environment becomes stable and the external excitation is stopped, the system will return to a dynamic equilibrium state. In the Auger process, the recombination of conduction band electrons and valence band holes transfers energy to the third carrier through the collision process. When electrons are excited by light or heat, the electrons absorb energy, so the entire particle is excited to a higher energy level ${ }^{21,22}$. When the electrons return to the ground state, the particles return to the ground state, thereby converting the excess energy into thermal energy that spreads inside the BLT-IENG (Supplementary Fig. 11b).

\section{Power generation performance of the BLT-IENG in the marine environment}

After determining that the BLT-IENG has the best water evaporation properties, we improved its power generation performance by varying marine environmental factors. Interestingly, unlike the common triboelectric nanogenerators (TENGs) generating an alternating current, our BLTIENG outputs a direct current. By increasing the external light to $2 \mathrm{~kW} \cdot \mathrm{m}^{-2}$, the total input power 
of the external environment is increased, which greatly improves the water evaporation rate of the BLT-IENG. Therefore, it has a higher open-circuit voltage $(0.894 \mathrm{~V})$, as shown in Fig. 4a, and a higher short-circuit current $(230.2 \mu \mathrm{A})$, as shown in Fig. 4b. After connecting a variable load (0$10^{6} \Omega$ ), its maximum power density is $12.86 \mu \mathrm{W} \cdot \mathrm{cm}^{-2}$ (Fig. 4c). Fig. $4 \mathrm{~d}$ shows that the open-circuit voltage of the BLT-IENG in sea water is increased to $1.01 \mathrm{~V}$, and it has a larger short-circuit current $(215.6 \mu \mathrm{A})$ in Fig. 4e, due to the ion concentration increase. However, when the ion concentration is too high, thus blocking the pores in the mesoporous BLT-IENG during the interfacial evaporation process, the water evaporation rate decreases. At the same time, the increase in the ion concentration increases the number of ions crystallized inside the BLT-IENG, thereby increasing its resistance and reducing its short-circuit current. Comparison studies show that the power generation performance of the BLT-IENG in sea water is better than that in the other solutions overall. After connection to various loads $\left(0-10^{6} \Omega\right)$, the maximum power can reach $13.61 \mu \mathrm{W} \cdot \mathrm{cm}^{-2}$ (Fig. $\left.4 \mathrm{f}\right)$. To further improve the power generation of the BLT-IENG, we change the wind speed on the surface of the BLT-IENG. Fig. 4g shows that the open-circuit voltage of the BLT-IENG increases to 1.106 $\mathrm{V}$ and its short-circuit current increases to $222.067 \mu \mathrm{A}$ under a wind speed of $1 \mathrm{~m} \cdot \mathrm{s}^{-1}$ (Fig. 4h). After connection to various loads $\left(0-10^{6} \Omega\right)$, the maximum power density can reach $15.46 \mu \mathrm{W} \cdot \mathrm{cm}^{-}$

2 (Fig. 4i). The increase in wind speed can effectively increase the water evaporation rate of the BLT-IENG. However, when the wind speed is too high, part of the water molecules is subjected to excessive force from the wind, so that they cannot interact with the BLC-layer and directly diffuse into the air. Although the increase in wind speed can increase the water evaporation rate of the BLTIENG, the ultra-high wind speed can also reduce the effective evaporation rate of the BLT-IENG for the power generation, thereby significantly weakening the power generation performance. Studies have shown that our BLT-IENG in sea water has the best power generation performance under a light intensity of $2 \mathrm{~kW} \cdot \mathrm{m}^{-2}$ and a wind speed of $1 \mathrm{~m} \cdot \mathrm{s}^{-1}$. It has a maximum open-circuit voltage of $1.106 \mathrm{~V}$, a maximum short-circuit current of $222.067 \mu \mathrm{A}$ and an output power of 245.61 $\mu \mathrm{W}$ without an external load.

\section{Working principle induced by interfacial evaporation and application of the BLT-IENG}

The above advantages of the BLT-IENG are mainly attributed to the following reasons (Fig. 5a). Above all, the bionic light-trapping structure reduces the angle of the incident light, so the incident light is reflected multiple times on the photothermal interface of the BLC-layer, increasing the light absorption efficiency and water evaporation area. The light-absorbing particles on the surface of the bionic light-trapping can transfer and convert photons multiple times. Therefore, this structure can increase the optical path and improve the light absorption efficiency. Meanwhile, the ITW- 
layer with $\mathrm{TiO}_{2}$ and $\mathrm{SiO}_{2}$ can reflect heat radiation, thereby forming a thermal insulation layer dispersed in the interior. This characteristic can improve the overall heat preservation effect of the BLT-IENG and reduce heat loss to improve its energy conversion efficiency. After the BLT-IENG undergoes phase-separation treatment, the internal $\mathrm{Cl}^{-}$is replaced. There are a large number of free $\mathrm{BMIM}^{+}$inside the BLT-IENG. This increases its internal ion concentration and the concentration difference with sea water. The capillary water absorption capacity of the BLT-IENG is improved compared with the original capillary adsorption function. At the same time, the introduction of wind can promote the loss of water molecules by increasing the airflow rate on the surface of the BLTIENG to increase the water evaporation rate and thus improve its power generation performance.

After that, as the carboxyl and hydroxyl groups of MWNTs have strong adsorption to water molecules, the anions $\left(\mathrm{OH}^{-}\right)$in the water molecules are adsorbed. Therefore, the bottom surface of MWNTs in contact with water is negatively charged. Cations $\left(\mathrm{H}_{3} \mathrm{O}^{+}\right)$in the water are attracted to the solid-liquid boundary and accumulate at the outlet of the microchannel due to the Coulomb force. Eventually, combined with the electric double layer theory, a potential difference is formed on both sides of the MWNT-microchannels in the evaporation interface. Because relative displacement occurs between MWNTs and water under the effect of interface evaporation, a pressure difference is generated between the entrance and exit of the MWNT-microchannels. It drives water molecules to pass through the MWNT-microchannels, which have negatively charged surfaces. In terms of quantity, the numbers of anions and cations are not equal within the Debye radius of the electric double layer, but they gradually approach the bulk phase values beyond the Debye radius. When water passes through the external pores, the ratio of anions and cations contained in the main part of the channel center is basically the same as that in the bulk phase. Therefore, there will be no difference in the ion concentrations on both sides of the MWNTmicrochannels, and the BLT-IENG appears to be electrically neutral. However, when water molecules pass through the MWNT-microchannels, which are narrow enough to overlap the electric double layer, the MWNT-microchannels will have ion-selective permeability. As a result, the flux of cations through the MWNT-microchannels will be larger than that of anions, and the solution in the MWNT-microchannels will no longer be electrically neutral. Moreover, cations and anions will accumulate at the exit and entrance of the MWNT-microchannels, respectively, thereby forming a flowing potential of the electrokinetic phenomenon.

At last, some anions and cations inside and outside the MWNT-microchannels can be removed by vapor during the interfacial evaporation, and the number of ions passing will decrease, thereby reducing the potential of the electric double layer. However, more water molecules participate in the interfacial evaporation are changed into vapor in the initial stage. Thus, there are more 
interfacial ions than those removed by vapor, and the voltage has a rising tendency at this moment. As time passes, the evaporation rate of the BLT-IENG gradually increases, and the pressure difference between the entrance and exit of the MWNT-microchannels increases. This results in the numbers of anions and cations produced by water molecules and MWNTs gradually increasing at the entrance and exit of the MWNT-microchannels, thereby gradually increasing the streaming potential. With the water evaporation rate reaching a stable value, anions and cations continuously accumulate at the entrance and exit of the MWNT-microchannels, and the number of anions and cations removed by vapor tends to stabilize, thereby forming a stable and maximum streaming potential. However, because no external circuit is connected, there is only a potential, and no current is formed. After the circuit of the two ends of the BLT-IENG is connected, electrons move along the wire from the wet end at the bottom to the dry end exposed to air via the action of the phonon wind and the fluctuating Coulomb field ${ }^{21}$, and a phonon-Coulomb field coupling direct current is generated (Fig. 5a).

Through the comparison of the evaporation performance under simulated marine environment, the water evaporation rate of the proposed BLT-IENG with sea water increases to $4.385 \mathrm{~kg} \mathrm{~m}^{-2} \mathrm{~h}^{-1}$ (increased nearly twice) at a $2.0 \mathrm{~kW} \cdot \mathrm{m}^{-2}$ light intensity and a $1 \mathrm{~m} \mathrm{~s}^{-1}$ wind speed (Fig. 5b). The produced fresh water can fully meet the WHO's requirements for human drinking standards (Supplementary Fig. 12a) ${ }^{25}$. The produced fresh water in the BLT-IENG is proven to be derived from sea water instead of the water inside the ITW-layer, further certifying the sustainability of our BLT-IENG (Supplementary Fig. 13). Moreover, our BLT-IENG can generate a maximum opencircuit voltage of $1.106 \mathrm{~V}$ and a maximum short-circuit current of $222.067 \mu \mathrm{A}$. After various external resistances $\left(0-10^{6} \Omega\right)$ are loaded in the circuit (Supplementary Fig. 12b), it still exhibits excellent power generation performance. When the load resistance reaches $4980 \Omega$, its loaded output power reaches a maximum of $61.85 \mu \mathrm{W}$. Compared with the average voltage per unit area of reported solar-driven TENGs $\left(36 \mathrm{mV} \mathrm{cm}^{-2}\right)^{26-34}$, our BLT-IENG $\left(276 \mathrm{mV} \mathrm{cm}^{-2}\right)$ is improved by a factor of 7.6 34.5 (average value smallest value) over that reported for solar-driven TENGs (Fig. 5 c) ${ }^{30}$.

The current generated by the BLT-IENG varies with its water evaporation rate. When the water evaporation rate of the BLT-IENG gradually increases to a stable value, the number of anions and cations rubbing against the MWNT molecules gradually increase and then stabilize. The output current of the BLT-IENG increases and then stabilizes. With the increase in the number of anions and cations passing through the MWNT-microchannels, the streaming potential increases obviously as anions and cations continuously accumulate at the entrance and exit of the MWNTmicrochannels. In this way, it can be proven that the improvement of the water evaporation rate can 
increase the number of water molecules that viscous slip along the MWNTs, which produces more $\mathrm{H}_{3} \mathrm{O}^{+}$and $\mathrm{OH}^{-}$, thereby generating larger current and voltage. In addition, the water flow rate theoretically has an exponential relationship with the open-circuit voltage and the short-circuit current of the BLT-IENG ${ }^{35}$. The relationship between the electron velocity and the solution flow rate is given see Supplementary Note 5 and Supplementary Note 6. From the equations, it can be concluded that we can obtain greater power generation performance by increasing the evaporation capacity and water evaporation rate of the BLT-IENG.

In addition, we designed a self-powered electronic integrated system (Fig. 5d). After simple signal processing, this integrated system can drive some low-voltage devices by absorbing solar and wind energy (Fig. 5e for working system circuit). Therefore, our BLT-IENG can provide time reminders for the marine equipment, and we can also collect electricity conveniently by charging batteries. In this way, this device can also be widely deployed on the sea for the freshwater production and the electric energy harvesting (Supplementary Fig. 14), which creates an opportunity to develop the offshore power generation platforms and the fresh water supply devices.

\section{Conclusion}

In summary, we demonstrated a solar and wind natural energies driven interfacial evaporation nanogenerator (BLT-IENG) including a hierarchical surface of bionic light-trapping and electrospinning perovskite conductivity with an enhanced thermally insulating and water storage capability. Meanwhile, we explained the coupling enhancement effect on interfacial evaporation of the BLT-IENG, and revealed the working principle of the power generation performance enhancement induced by interfacial evaporation. Hence, our BLT-IENG can achieve an excellent power generation performance of an open-circuit voltage of $1.106 \mathrm{~V}$, a short-circuit current of $222.07 \mu \mathrm{A}$, a unit area voltage of $276 \mathrm{mV} \mathrm{cm}^{-2}$ and an output power of $245.61 \mu \mathrm{W}$ under a 2.0 $\mathrm{kW} \cdot \mathrm{m}^{-2}$ light intensity and a $1 \mathrm{~m} \mathrm{~s}^{-1}$ wind speed. The working principle of the BLT-IENG can be summarized as the phonon-Coulomb coupling direct current under the coupling action of the phonon wind and a fluctuating Coulomb field. This work demonstrates an interfacial evaporation inspired sustainable spontaneously power generation technology and provides a foundation for the multiple utilization of natural energies. Meanwhile, it can also generate an opportunity to develop the offshore power generation platforms and the fresh water supply devices.

\section{Methods}

Materials. The ionic liquid of 1-n-butyl-3-methylimidazolium chloride (BMIMCl) used in the experiment was purchased from Lanzhou Institute of Chemical Physics, Chinese Academy of Sciences. $\mathrm{TiO}_{2}$, TiNx, polyethylene glycol (PET, molecular weight $100 \mathrm{MW}$ ), and $\mathrm{CsPbBr}_{3}$ and 
$\mathrm{Cs}_{4} \mathrm{PbBr}_{6}$-type perovskites were purchased from Macleans Co., Ltd. $\alpha$-cellulose (90 $\mu \mathrm{m}$ particle size) and chitosan (Cs) were obtained from Aladdin Co., Ltd. Silica ( $\mathrm{SiO}_{2}$, nanoscale) was purchased from Scientific Industrial Co., Ltd. Acetic acid (purity $60 \%$ ) was purchased from China Harbin Yongchang Reagent Co., Ltd. MWNTs (diameter $15 \mathrm{~nm}$ ) were purchased from Turing Technology Co., Ltd.

Preparation process of the BLT-IENG. Chitosan (Cs) was dissolved in $2 \%$ HAc solution under water bath conditions. PET, $\mathrm{Cs}_{4} \mathrm{PbBr}_{6}$ and an MWNT aqueous dispersion (concentration of $10 \mathrm{wt} \%$ ) were added in sequence. Then, the solution was stirred and injected into a JDF05 electrostatic spinning machine. Finally, $\mathrm{Cs}_{4} \mathrm{PbBr}_{6}$ and a conductive layer were constructed on the upper and lower surface with bionic moth eye structure. $\alpha$-cellulose was dissolved in BMIMCl under water bath conditions. $\mathrm{TiO}_{2} / \mathrm{SiO}_{2} / \mathrm{TiN}_{\mathrm{x}} / \mathrm{MWNTs}$ were added to the solution in sequence and stirred evenly. Then, the solution was placed and cooled in a common mold.

Materials characterization. The structure characteristics of the BLT-IENG were observed through a scanning electron microscope with an accelerating voltage of $5 \mathrm{kV}$ (JSN-7500F, Japan) and a transmission electron microscope with an accelerating voltage of $200 \mathrm{kV}$ (JEM-2100, Japan). The optical transmittance and the reflectance spectrum of the BLT-IENG were measured with an ultraviolet-visible spectrophotometer (Lambda 950, USA) in the range of 190-3000 nm. The light absorption efficiency was calculated by the equation $\mathrm{A}=1-\mathrm{R}-\mathrm{T}$, where $\mathrm{R}$ and $\mathrm{T}$ are the reflection and transmission efficiencies of the BLT-IENG, respectively. Infrared photos of the BLT-IENG were taken by an HT-18 infrared camera. The evaporation experiment was carried out under a solar simulator of a CEL-SA500/350 xenon lamp. The ion concentration was tested using an ICP-OES inductively coupled plasma spectrometer and an ICS-600 ion chromatograph instrument. The power generation performance of the BLT-IENG was measured by a Keithley 6514 electrometer (USA).

\section{Data availability}

The data that support the plots within this paper and other findings of this study are available from the corresponding author upon reasonable request.

\section{References}

1. Peterson, T. C., Golubev, V. S. \& Groisman, P. Y. Evaporation losing its strength. Nature, 1995, 377(6551): 687688.

2. Brutsaert, W., \& Parlange, M. B. Hydrologic cycle explains the evaporation paradox. Nature, 1998, 396(6706): 3030.

3. Xue, G. et al. Water-evaporation-induced electricity with nanostructured carbon materials. Nat. Nanotechnol. 12,317-321(2017).

4. Xu, N. et al. A water lily-inspired hierarchical design for stable and efficient solar evaporation of high-salinity brine. Sci. Adv. 5, eaaw7013 (2019).

5. Geng, H. et al. Plant leaves inspired sunlight-driven purifier for high-efficiency clean water production. Nat. Commun., 2019, 10(1): 1-10. 
6. Tao, P. et al. Solar-driven interfacial evaporation. Nature Energy 3, 1031-1041 (2018).

7. Lu, Z. et al. A unified relationship for evaporation kinetics at low Mach numbers. Nat. Commun. 10, 1-8 (2019).

8. Liu, Q. et al. Tree frog-inspired micropillar arrays with nanopits on the surface for enhanced adhesion under wet conditions. ACS Appl. Mater. Inter. 12, 19116-19122 (2020).

9. Ma, C. et al. Bioinspired anisotropic hydrogel actuators with on-off switchable and color-tunable fluorescence behaviors. Adv. Funct. Mater. 2018, 28(7): 1704568.

10. Li, Q. et al. Reversible structure engineering of bioinspired anisotropic surface for droplet recognition and transportation. Adv. Sci. 7, 2001650 (2020).

11. Le, X., Lu, W., Zhang, J. \& Chen, T. Recent progress in biomimetic anisotropic hydrogel actuators. Sci. Adv. 6, 1801584 (2019).

12. Han, Z. et al. Ren, Light trapping structures in wing scales of butterfly trogonoptera brookiana. Nanoscale 4, 28792883 (2012).

13. Suter, S., Graf, R., Garcia, D. M. \& Haussener, S. Optimizing and implementing light trapping in thin-film, mesostructured photoanodes. ACS Appl. Mater. Inter. 12, 5739-5749 (2020).

14. Han, Z. et al. An ingenious replica templated from the light trapping structure in butterfly wing scales. Nanoscale 5, 8500-8506 (2013).

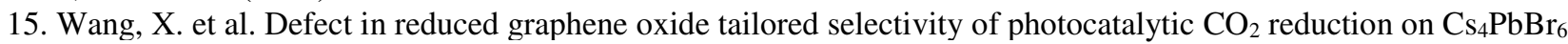
pervoskite hole-in-microdisk structure. Nano Energy 78, 105388 (2020).

16. Zhang, W. et al. Submillimeter-scale zero-dimensional $\mathrm{Cs}_{4} \mathrm{PbBr}_{6}$ perovskite rods: fabrication, optical properties, and applications. ACS Appl. Mater. Inter. 2, 2408-2417 (2020).

17. Hu, X. \& Zhu, J. Tailoring aerogels and related 3d macroporous monoliths for interfacial solar vapor generation. Adv. Funct. Mater. 30, 1907234 (2020).

18. Sun, Z. et al. A high-efficiency solar desalination evaporator composite of corn stalk, $\mathrm{Mcnts}_{\text {and }} \mathrm{TiO}_{2}$ : ultra-fast capillary water moisture transportation and porous bio-tissue multi-layer filtration. J. Mater. Chem. A 8, 349-357 (2020).

19. Sun, W. et al. Han, Wind energy harvesting based on fluttering double-flag type triboelectric nanogenerators. Nano Energy 70,104526 (2020).

20. Beni, A. A. \& Esmaeili, A. Fabrication of 3D hydrogel to the treatment of moist air by solar/wind energy in a simulated battery recycle plant salon. Chemosphere 246, 125725 (2020).

21. Liu, A. et al. Strano, Direct electricity generation mediated by molecular interactions with low dimensional carbon materials-A mechanistic perspective. Adv. Energy Mater. 8, 1802212 (2018).

22. Kovalenko, M. V. et al. Prospects of nanoscience with nanocrystals. ACS Nano 9, 1012-1057 (2015).

23. Dong, T. et al. Study on bionic moth eye antireflective cylindrical microstructure on germanium substrate. Acta Optica Sinica 36, 236-242 (2016).

24. Zhao, S., Jensen, O. M. \& Hasholt, M. T. Measuring absorption of superabsorbent polymers in cementitious environments. Mater. Struct. 53, 11 (2020).

25. Edition, F. Guidelines for drinking-water quality. WHO Chron. 38, 104-108 (2011).

26. Dao, V. D., Vu, V. H. \& Choi, H. S. All day limnobium laevigatum inspired nanogenerator self-driven via water evaporation. J. Power Sources 448, 227388 (2020).

27. Das, S. S. et al. Electrical power generation from wet textile mediated by spontaneous nanoscale evaporation. Nano Lett. 19, 7191-7200 (2019).

28. Hou, B. et al. Flexible graphene oxide/mixed cellulose ester films for electricity generation and solar desalination. Appl. Therm. Eng. 163, 114322 (2019).

29. Hou, B. et al. Flexible and portable graphene on carbon cloth as a power generator for electricity generation. Carbon 140, 488-493 (2018).

30. Xiao, P. et al. Exploring interface confined water flow and evaporation enables solar-thermal-electro integration towards clean water and electricity harvest via asymmetric functionalization strategy. Nano Energy 68, 104385 (2020).

31. Sun, J. et al. Electricity generation from a Ni-Al layered double hydroxide-based flexible generator driven by natural water evaporation. Nano Energy 57, 269-278 (2019).

32. Zhang, G. et al. Harvesting environment energy from water-evaporation over free-standing graphene oxide sponges. Carbon 148, 1-8 (2019).

33. Li, L. et al. Sustainable and flexible hydrovoltaic power generator for wearable sensing electronics. Nano Energy 72, 104663 (2020).

34. Ding, T. et al. All-printed porous carbon film for electricity generation from evaporation-driven water flow. Adv. Funct. Mater. 27, 1700551 (2017).

35. Liu, A. T. et al. Direct electricity generation mediated by molecular interactions with low dimensional carbon materials-A mechanistic perspective. Adv. Energy Mater. 8, 1802212 (2018). 


\section{Acknowledgments}

This work is supported by China Postdoctoral Science Foundation Funded Project (Grant No. 2018 M630330 \& No. 2019T120245), National Natural Science Foundation of China (Grant No. 51905085)

\section{Author contributions}

Z.S. conceived the idea. Z.L. designed the experiment and analyzed the data, Z.S., C.H., M. J. provide financial support. H.Y. and Z. W. provided technical support for cellulose and experiments. Z.S., Z.L. wrote the article, and Z.W., Z.S. conducted the final review of this article. All authors discussed the results and commented on the manuscript.

\section{Competing interests}

The authors declare that they have no competing interests. 
Figures and Tables

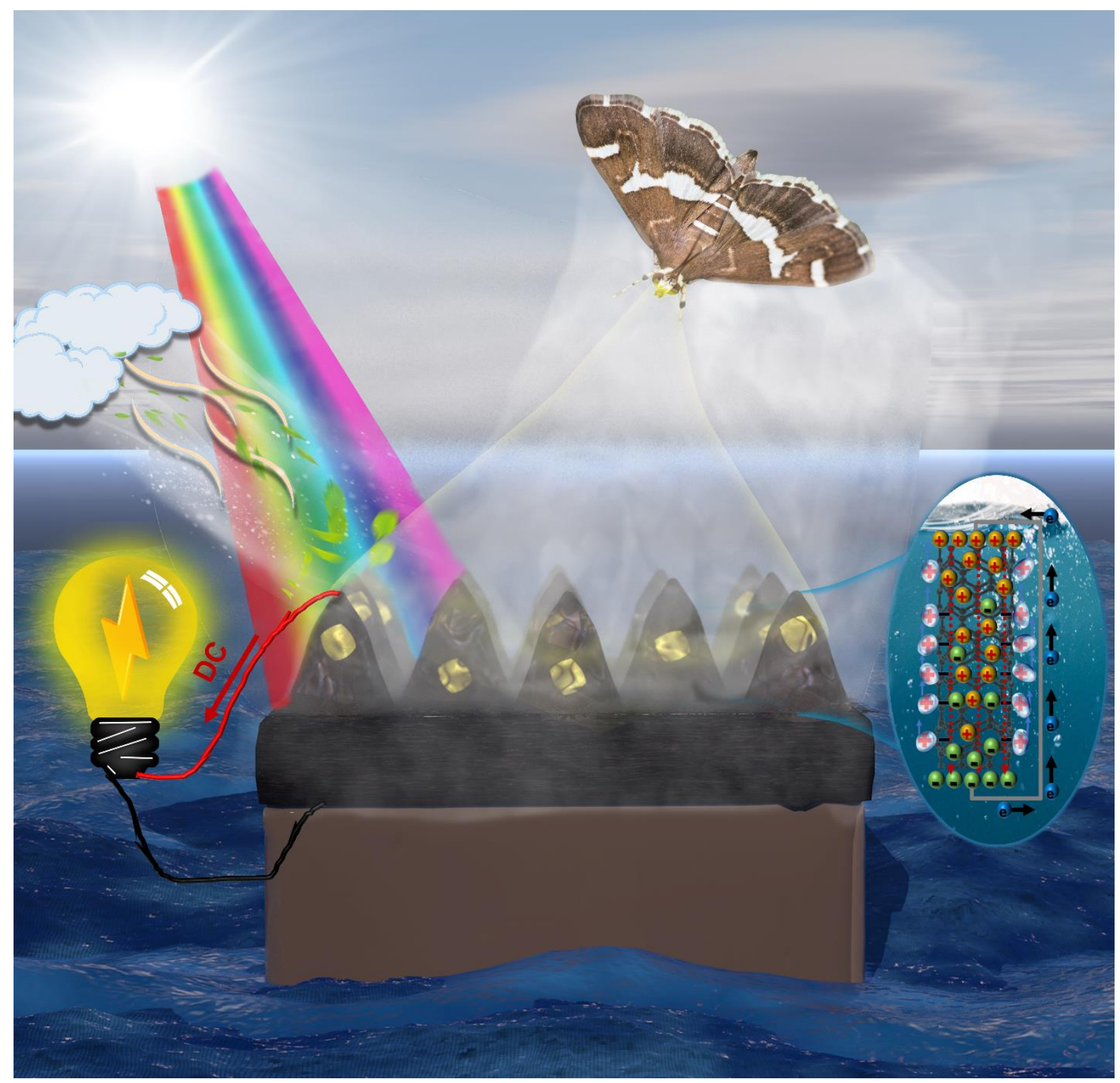

Title page of bioinspired interfacial evaporation driven nanogenerator. 
a
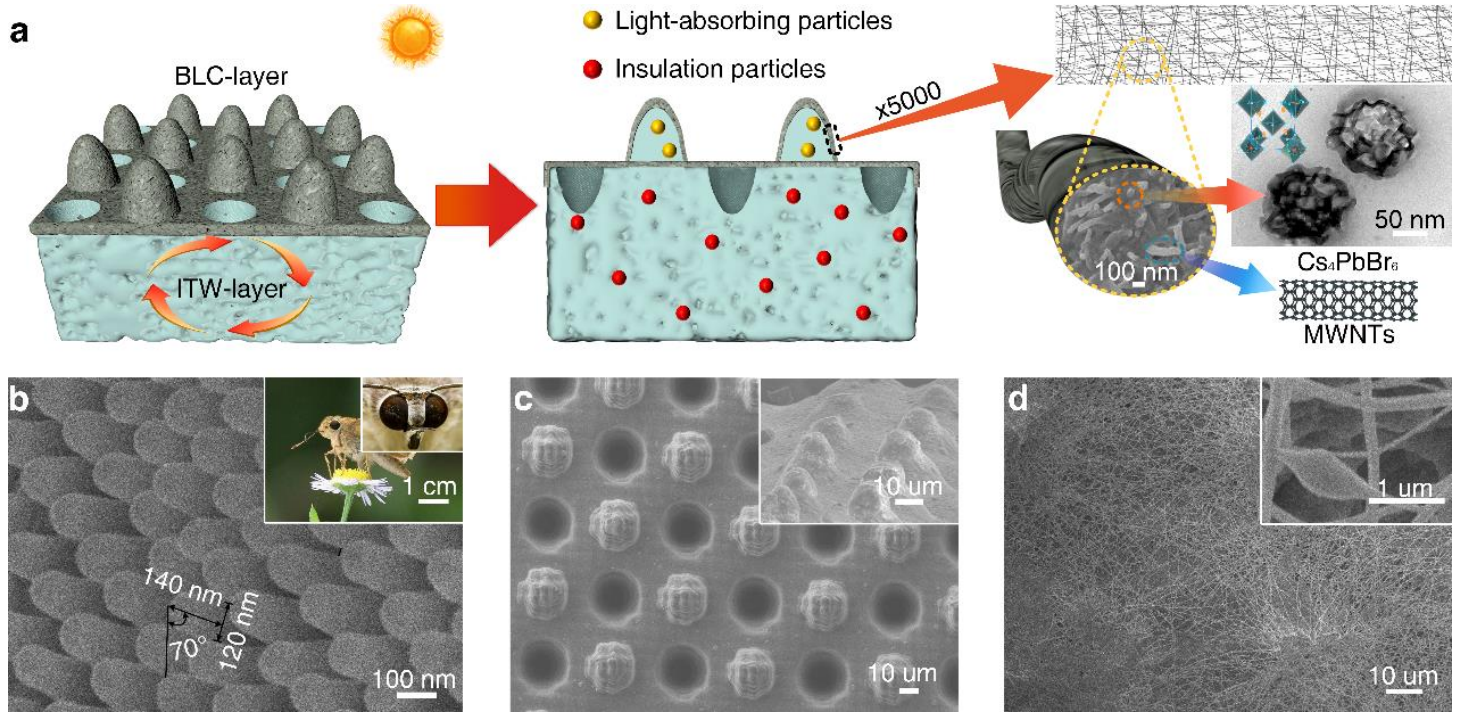

Fig. 1. Structure and characterization of the BLT-IENG. a Structural design and composition of the BLT-IENG.

b Morphological characteristics of the moth eye. c Surface structure of the fabricated bionic moth eye of the BLClayer. d The surface microstructure of the electrospinning BLC-layer. 

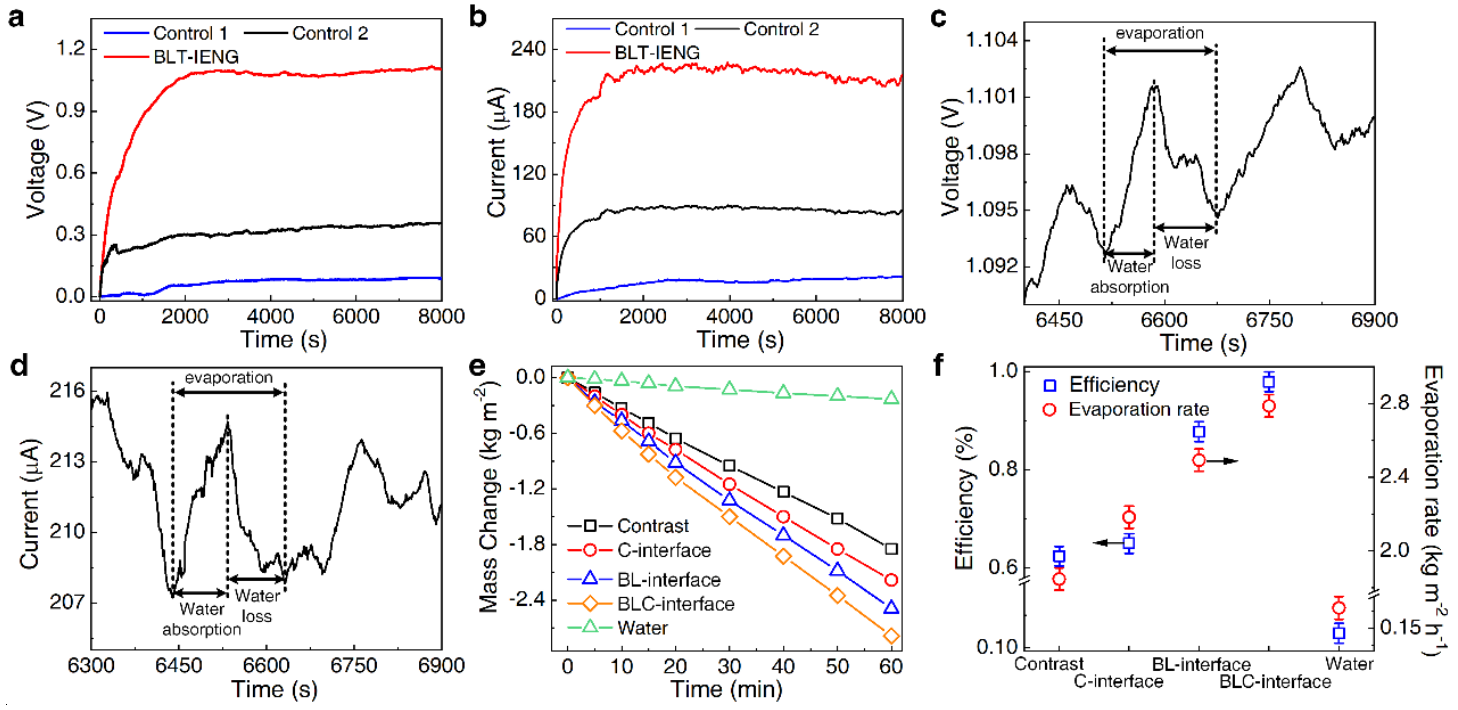

Fig. 2. Power generation performance and evaporation characteristics of the BLT-IENG. a The open-circuit voltage of the BLT-IENG, Control 1 ( $\alpha$-cellulose hydrogels with $\mathrm{TiO}_{2}, \mathrm{SiO}_{2}$ and $\mathrm{TiNx}$ ) and Control 2 ( $\alpha$-cellulose hydrogels with $\mathrm{TiO}_{2}, \mathrm{SiO}_{2}$, TiNx and MWNTs). b The short-circuit current of the BLT-IENG, Control 1 and Control 2. $\mathbf{c}$ The open-circuit voltage of the BLT-IENG during water evaporation. $\mathbf{d}$ The short-circuit current of the BLTIENG during water evaporation. e Evaporation rates of BLT-IENGs in $1 \mathrm{~h}$. f Energy conversion efficiencies and water evaporation rates of BLT-IENGs. Here, C-interface is the perovskite conductivity interface and BL-interface is the bionic light-trapping interface. 

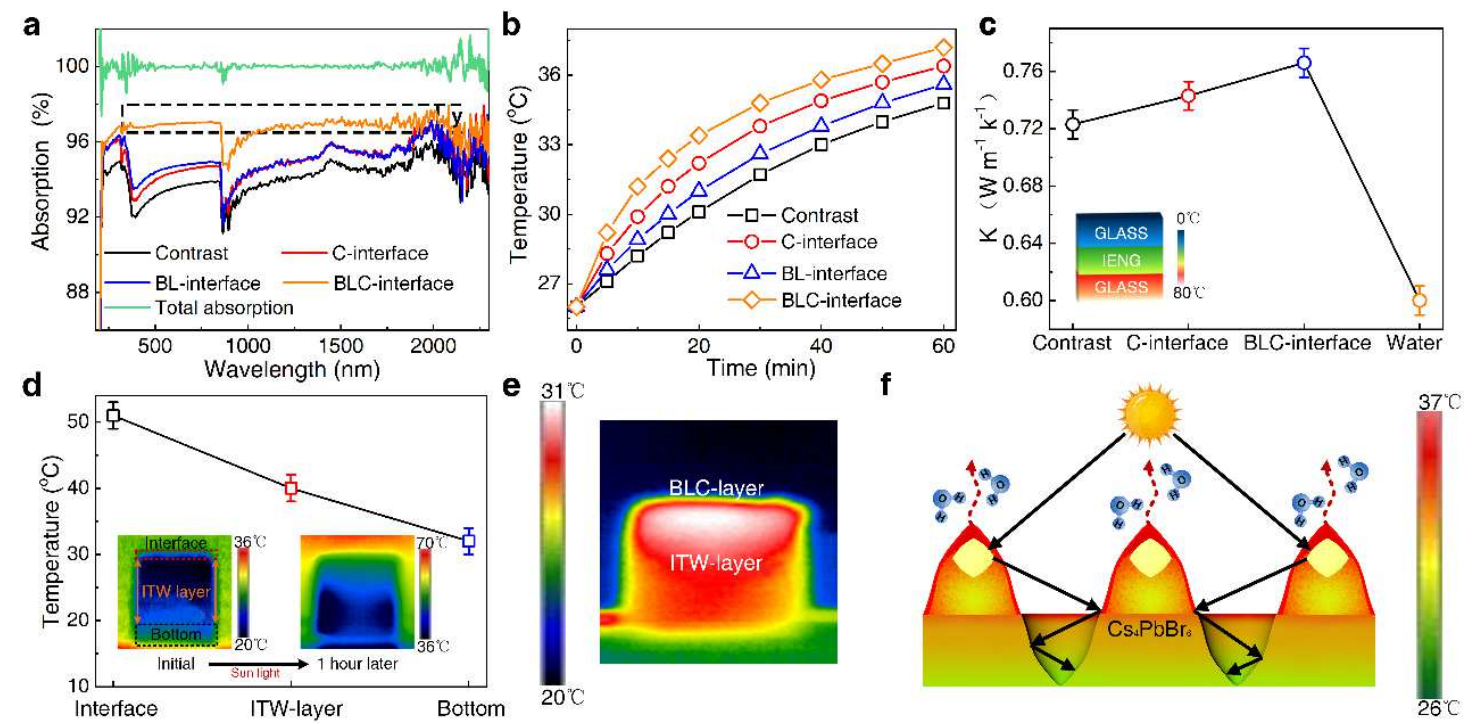

Fig. 3. Photothermal conversion characteristics of the BLT-IENGs. a Reflectance absorption spectrum of BLTIENGs in the wavelength range of 190-2500 $\mathrm{nm}$. b Surface temperature curves of BLT-IENGs under a solar light intensity of $1.0 \mathrm{~kW} \cdot \mathrm{m}^{-2}$. $\mathbf{c}$ Thermal conductivity of the BLT-IENGs with the BLC-layer. $\mathbf{d}$ Temperature gradient of BLT-IENGs during water evaporation. e Temperature gradient of BLT-IENG placed $20 \mathrm{~min}$ in the natural environment after illuminated by the sunlight for $1 \mathrm{~h}$. $\mathbf{f}$ Improvement principle of interface photothermal conversion of bionic moth eye structure. Here, C-interface is the perovskite conductivity interface and BL-interface is the bionic light-trapping interface. 
a
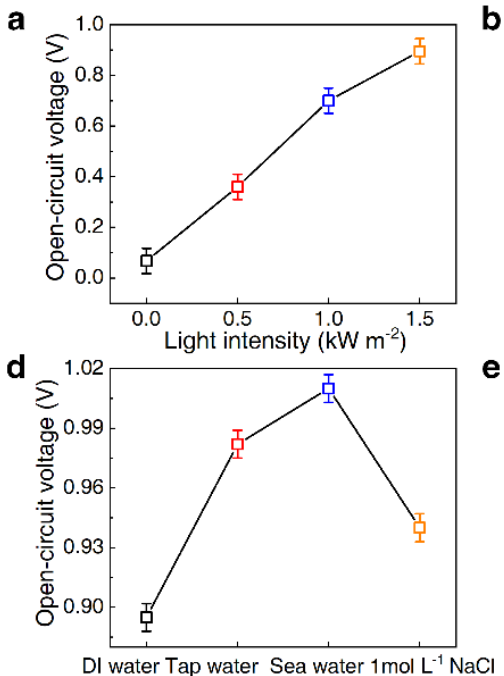

g

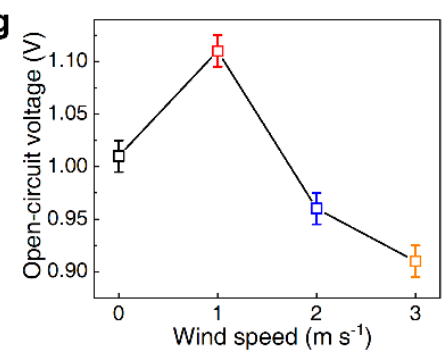

h b
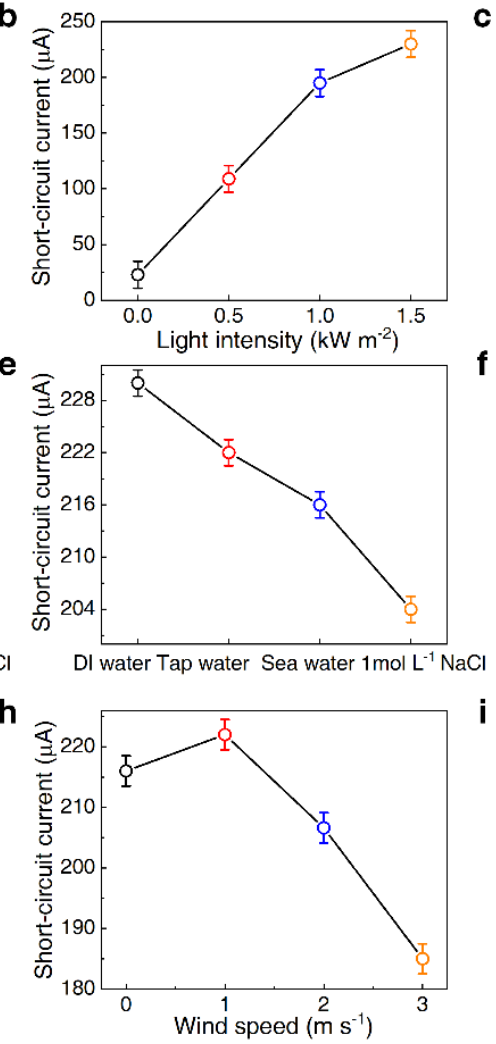

c
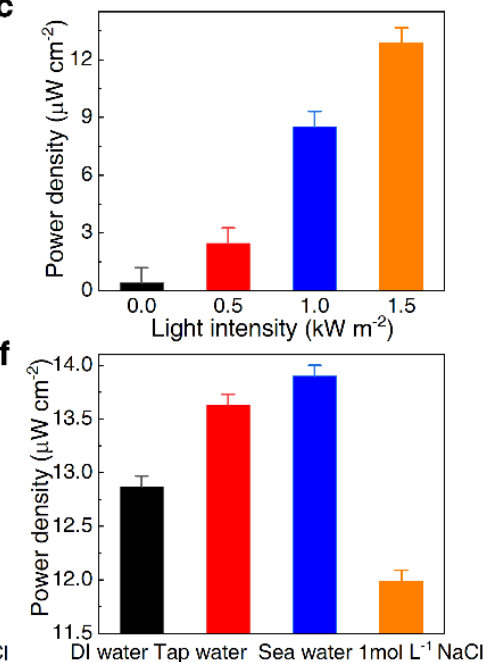

i

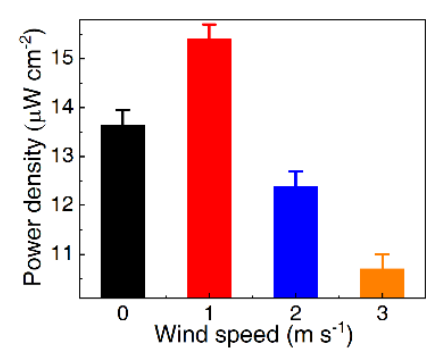

Fig. 4. Power generation performance of the BLT-IENG under different surrounding conditions. a-c Power generation performance of the BLT-IENG with the ITW layer under different light intensities. d-f Power generation performance of the BLT-IENG with the ITW layer in different types of liquid. g-i Power generation performance of the BLT-IENG under different wind speeds. 

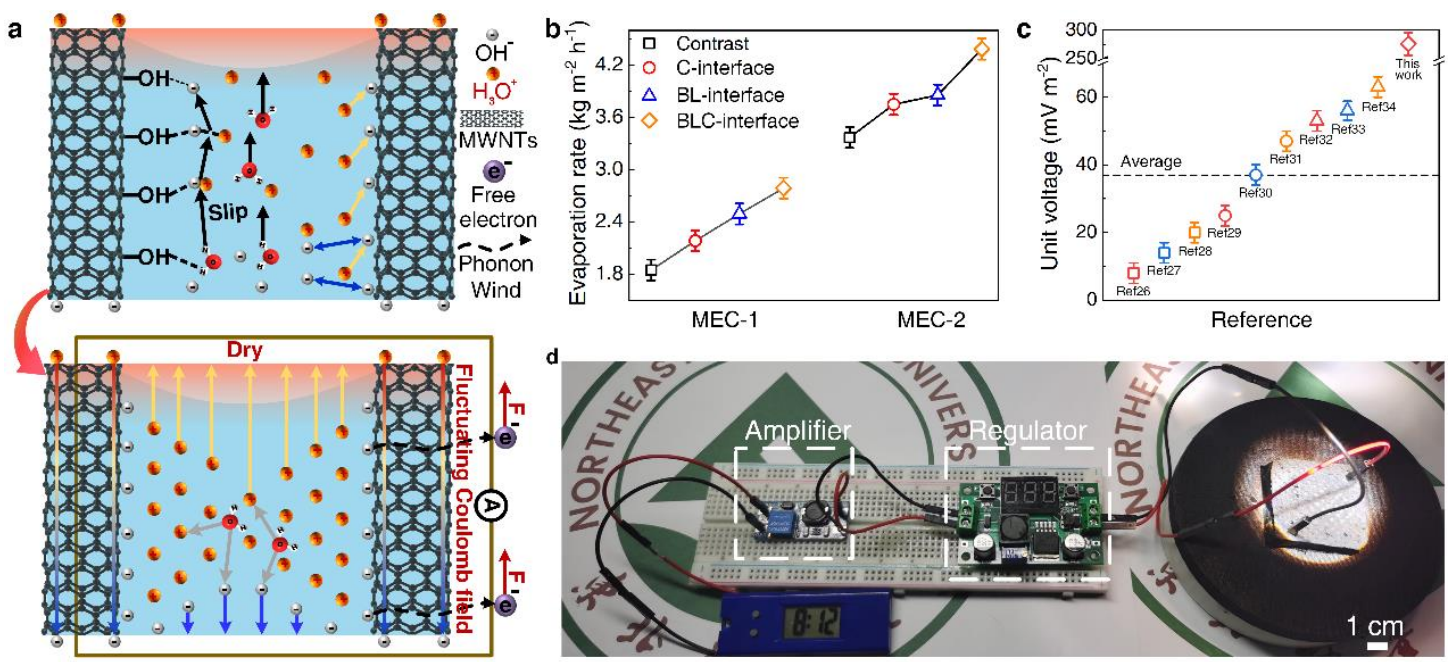

e

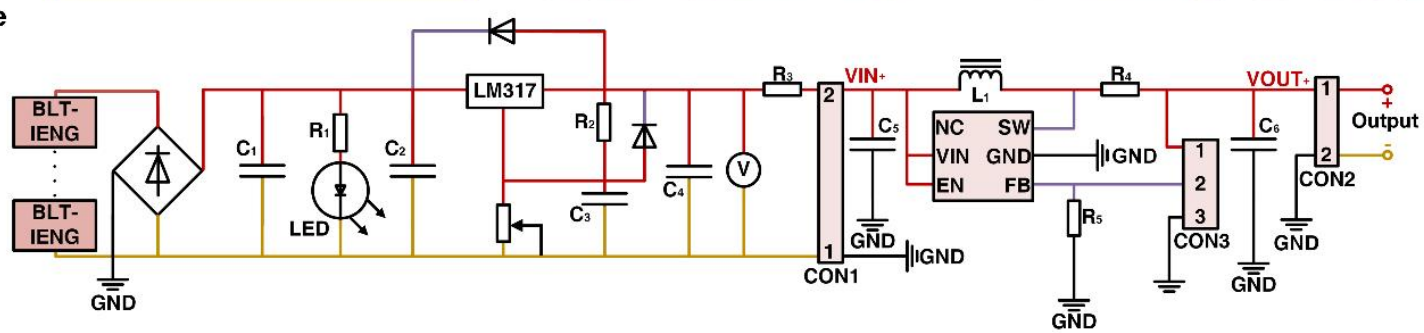

Fig. 5. Working principle and application of the BLT-IENG. a Power generation working principle of the BLTIENG induced by water interfacial evaporation. b Water evaporation rate of BLT-IENGs under simulated marine environment. Marine environmental condition 1 (MEC-1) of a $1.0 \mathrm{~kW} \cdot \mathrm{m}^{-2}$ light intensity, no wind speed and deionized water and marine environmental condition 2 (MEC-2) of a $2.0 \mathrm{~kW} \cdot \mathrm{m}^{-2}$ light intensity, a $1 \mathrm{~m} \mathrm{~s}^{-1}$ wind speed and sea water. c Comparison of voltages per unit area generated by the BLT-IENG and other solar-driven evaporators. d Photograph of the self-powered working system. e Schematic diagram of the self-powered working system circuit. Here, C-interface is the perovskite conductivity interface and BL-interface is the bionic light-trapping interface. 


\section{Supplementary Files}

This is a list of supplementary files associated with this preprint. Click to download.

- SupplementaryMaterials2021724.pdf 